

\title{
Editorials
}

\section{Dynamic navigation system: A real time guide to endodontists}

\author{
Vineeta Nikhil ${ }^{1, *}$ \\ ${ }^{1}$ Dept. of Conservative Dentistry and Endodontics, Subharti Dental College, Meerut, Uttar Pradesh, India
}

A R T I C L E I N F O

Article history:

Received 12-03-2021

Accepted 14-03-2021

Available online 16-03-2021
(C) This is an open access article distributed under the terms of the Creative Commons Attribution License (https://creativecommons.org/licenses/by/4.0/) which permits unrestricted use, distribution, and reproduction in any medium, provided the original author and source are credited.
The management of calcific metamorphosis or pulp canal obliteration can be quite challenging for us as clinicians. Access cavity preparation and cleaning and shaping of such cases are likely prone to procedural errors that may lead to a substantial loss of sound dentin structure, thereby reducing the long-term prognosis. The use of CBCT and 3D printing has proved a boon to the clinician in every field of dentistry, especially Endodontics, which has led to the advent of guided endodontics. ${ }^{1}$ This has transformed the pretreatment planning as well as the management procedure completely. Through the use of this technology static navigation stents can be fabricated. These stents direct the access cavity preparation, thus avoiding the removal of sound tooth which is inadvertent while working freehanded. ${ }^{2}$

Another technical advancement in this field is the Dynamic Navigation Technology. It offers real-time navigation which is a viable alternative to statically guided endodontics. Dynamic navigation systems (DNSs) were originally introduced in dentistry in the US in 2000 to facilitate insertion of dental implants. ${ }^{3}$ There is potential for this technology to be used in the field of Endodontics as well. Dynamic navigation combines surgical instrumentation and radiologic images by using an optical positioning device controlled by a dedicated computerized interface. It eliminates the operator's error regarding the dependency on anatomical landmarks. The motion tracking in this system enables it to follow the position of both the

\footnotetext{
* Corresponding author.

E-mail address: endobyvineeta@gmail.com (V. Nikhil).
}

clinician and the patient. ${ }^{4}$

The working of DNS can be simplified into four steps: Firstly, a pretreatment CBCT scan is done and the data is fed to the DNS program software. Secondly,this CBCT data is used in the planning of the nonsurgical 3D virtual access opening trajectories. Thirdly, clinical tracing of the jaw with a tracer tool is done to register the CBCT scan to the patients jaw for navigation. A complete accuracy check is performed in all the three directions. At last, the dental drill is also calibrated and the navigated access cavity preparation is made. The progression and direction of the drill can be monitored on the target view provided. ${ }^{5}$

Although DNS has not been much applied in the field of Endodontics, it has been well established in limited number of studies conducted that use of DNS increases the benefits of a conservative preparation by minimizing the potential risk of weakening of critical portions of the crown and reducing negative influences to shaping procedures. ${ }^{6}$ Not only in nonsurgical endodontics, DNS finds application in microsurgical endodontic procedures as well. It allows the clinician to accurately locate the apex of the root and properly direct the bur, reducing the risk of unintentional iatrogenic damage to nearby anatomic structures and perform minimal invasive intervention or flapless surgery, leading to reduction in patient postoperative discomfort and improved healing. ${ }^{4}$

Minimal invasive protocols are the stepping stones to the future of endodontics. Although not much worked upon, DNS is a promising digital alternative management strategy. 
Early adoption of this strategy is osmotic, wider acceptance will occur through diffusion.

\section{Conflict of Interest}

None.

\section{References}

1. Sikri J, Sikri A. 3D Endodontics: The Future of Dentistry. J Dent Oral Sci . 2020;2(3):1-2. doi:10.37191/mapsci-2582-3736-2(3)-038.

2. Anderson J, Wealleans J, Ray J. Endodontic applications of 3D printing. Int Endod J. 2018;51(9):1005-18. doi:10.111/1ej.12917.

3. Pellegrino G, Bellini P, Cavallini PF, Ferri A, Zacchino A, Taraschi $\mathrm{V}$, et al. Dynamic Navigation in Dental Implantology: The Influence of Surgical Experience on Implant Placement Accuracy and Operating Time. An in Vitro Study. Int J Environ Res Public Health. 2020;17(6):2153-61. [oi:10.3390/ijerph17062153.

4. Gambarini G, Galli M, Stefanelli LV, Nardo DD, Morese A, Seracchiani M, et al. Endodontic Microsurgery Using Dynamic
Navigation System: A Case Report. J Endod . 2019;45(11):1397-402. doi:10.1016/j.joen.2019.07.010

5. Jain SD, Carrico CK, Bermanis I. 3-Dimensional Accuracy of Dynamic Navigation Technology in Locating Calcified Canals. $J$ Endod. 2020;46(6):839-45. doi:10.1016/1.joen.2020.03.014,

6. Gambarini G, Galli M, Morese A, Stefanelli LV, Abduljabbar F, Giovarruscio M, et al. Precision of Dynamic Navigation to Perform Endodontic Ultraconservative Access Cavities: A Preliminary In Vitro Analysis. $J$ Endod . 2020;46(9):1286-90. doi:10.1016/j.joen.2020.05.022.

\section{Author biography}

Vineeta Nikhil, Professor \& Head

Cite this article: Nikhil V. Dynamic navigation system: A real time guide to endodontists. IP Indian J Conserv Endod 2021;6(1):1-2. 